\title{
Comparative Evaluation of Edge Linking Methods Using Markov Chain and Regression Applied Heuristic
}

\author{
Haklin Kimm, Neda Abolhassani, and Eun-Joo Lee \\ Computer Science Department \\ East Stroudsburg University of Pennsylvania \\ East Stroudsburg, PA, USA \\ $\{$ hkimm, elee\}@po-box.esu .edu
}

\begin{abstract}
There have been many studies on how to develop accurate edge detection algorithms for various applications - especially processing geographic images and maps, say coastline images. In this paper, we present edge linking algorithms, based on a heuristic approach using regression analysis and Markov chain technique respectively, for the coastline images. The heuristic approach consists of costs that are based on the distance and direction of the edge terminators while the Markov chain technique has been developed to investigate possible line drawing options to reconnect the broken edges so that a Markov transition matrix is generated in order to find the suitable edge terminators that can be reconnected. In this paper both techniques of using Markov chain and regression analysis have been developed and their outcomes have been evaluated comparatively upon their accuracy of reconnecting the broken edges.
\end{abstract}

Keywords: Edge detection and linking, Markov Chains, Regression based heuristic, Image Processing, Coastline images.

\section{Introduction}

Edges are considered as one of the most significant features of an image. Many of the edge detectors work based on the gradient at an edge point and the direction of the edge on that point [1], [2]. Conventional edge detectors have some problems due to differentiation functions and noises in an image. The source for the noise problem in coastline detection of satellite images can be clouds or other objects that result in an unclear image of the position of ocean and nearby land area [3], [4]. There have been various edge detection algorithms such as Prewitt, Sobel, Canny and Morphological that have been applied to the coastal satellite images [5]. In this paper, we present edge linking algorithms based on a heuristic function using regression analysis and Markov chain technique respectively. The heuristic approach, say function, consists of costs which are based on the distance and direction of the edge terminators. The proposed heuristic function is explained in detail with its regression analysis technique in later section; and followed by Markov chain technique for reconnecting edges [6], [7].

Due to presence of non-continuous edges in the coastline images of edges, this work first implements an edge linking method which is based on Markov transition 
matrix. The Markov chain has been developed to investigate possible line drawing options to reconnect the broken edges and to verify whether the use of the Markov chain provide a proper pattern for a linking path between two candidate edge terminators. This work also presents another edge linking method using the regression based heuristic instead of Markov chain, which has been developed, tested, and measured of its success rate of reconnecting broken edges with the Markov chain approach. The Bresenham's line drawing algorithm, which is quite useful in computer graphics, has been applied upon the candidate edge terminators [8], [9], generated and recommended by both techniques- regression based and Markov applied respectively.

The rest of the paper is organized as follows. In Section 2, link pattern analysis using Markov Chain is presented with its implementations; in Section 3, edge linking heuristics based on regression analysis are introduced and implemented; in Section 4, the result images of both techniques are evaluated comparatively. Finally, the paper concludes with its summary.

\section{Edge Linking Based on the Markov Chain}

The Markov property is for the cases that the state of the system at time $t+1$ depends only on the state of the system at time t. The transition matrix shows us the probability of a specific direction of an edge segment after another one. In order to create the transition matrix, we need to get the states of the edge pixels' directions. The numbers of patterns that occur after a horizontal edge, vertical edge and two types of diagonal edges have to be counted. For example, if a(i,j) is the current pixel, any of the $a(i-1, j)$ or $a(i+1, j)$ can make a vertical edge. Any of the $a(i, j-1)$ or $a(i, j+1)$ can make a horizontal edge. Any of $a(i-1, j-1)$ or $a(i+1, j+1)$ can create a diagonal edge referred as dgn 1 edge in this paper and any of $a(i-1, j+1)$ or $a(i+1, j-1)$ can create a different diagonal edge referred as dgn2 edge. Equation 1 shows how we can calculate the probability of being a horizontal edge segment after a vertical edge segment Pverhor:

$$
\mathrm{P}_{\text {verhor }}=\frac{\text { the number of horizontel edges after vertical edges }}{\text { the total number of patterns that occur after vertical edges }}
$$

Figure 2 is one of the images that has analyzed for this edge linking pattern analysis problem using a Markov chain on the Figure 1(C). The following is the Markov transition matrix generated after processing the image over the MATLAB programs [10]:

\begin{tabular}{|c|c|c|c|c|}
\hline $\mathrm{P}=$ & hor & ver & $d g n 1$ & dgn 2 \\
\hline hor & {$[0.6311$} & 0.0410 & 0.2295 & 0.09847 \\
\hline ver & 0.0022 & 0.9307 & 0.0671 & 0 \\
\hline$d g n 1$ & 0.2885 & 0.3462 & 0.3462 & 0.0192 \\
\hline$d g n 2$ & Lo.3077 & 0.4103 & 0.1026 & [0.1795 \\
\hline
\end{tabular}




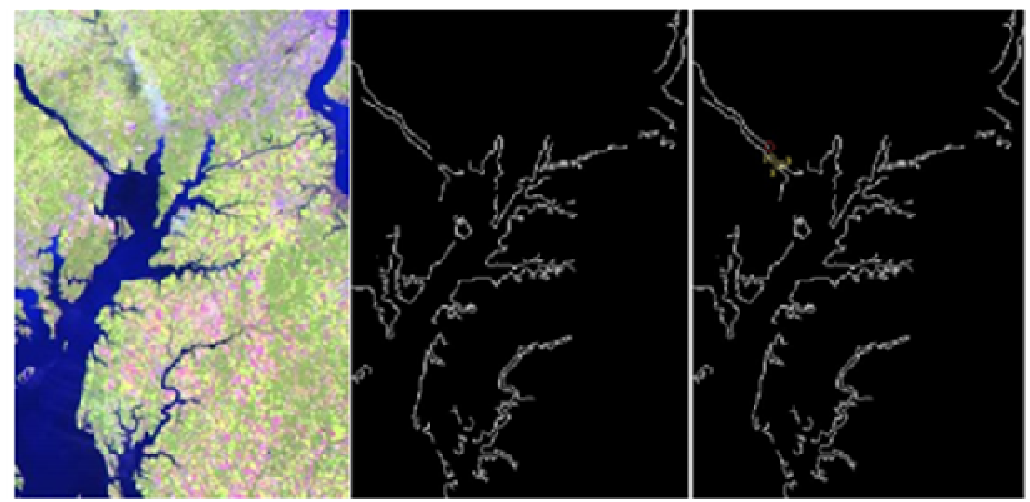

Fig. 1. Starting from the left (A): Original image (US East Coast), middle (B): Sobel edge detection result, and right $(\mathrm{C})$ : Detecting source and target endpoints



Fig. 2. Outcome image after applying Markov Chains to Figure 1(C)

The maximum probability of each pattern in each row can be selected as the best pattern for the next move, and they are highlighted in the above matrix. The probability of going from state $i$ to $j$ in $n$ steps can be found by the transition matrix raised to the power of $n$. In the edge linking problem $n$ can be the distance between the two endpoints that are going to be linked. For this case the shortest distance is 6 and the transition matrix should be raised from power of 2 to 6 , which are shown in the equations 2 thru 4.

$$
\begin{aligned}
& \mathrm{P}^{(2)}=\text { hor ver dgn1 dgn2 } \\
& \begin{array}{c}
\text { hor } \\
\text { ver } \\
\text { dgn1 } 1 \\
\text { dgn2 } 2
\end{array}\left[\begin{array}{llll}
0.4949 & 0.1838 & 0.2371 & 0.0841 \\
0.2886 & 0.8896 & 0.0862 & 0.0015 \\
0.2799 & 0.5036 & 0.1521 & 0.0645
\end{array}\right]
\end{aligned}
$$




\begin{tabular}{|c|c|c|c|c|}
\hline & $\mathbf{P}^{(\mathbf{3})}$ & $\cdots .$. & $\cdots .$. & $\mathbf{P}^{(5)}$ \\
\hline $\mathbf{P}^{(6)}$ & hor & ver & $d g n$ & $\operatorname{dgn} 2$ \\
\hline hor & {$[0.1109$} & 0.7541 & 0.1189 & $0.0161]$ \\
\hline ver & 0.1107 & 0.7545 & 0.1188 & 0.0161 \\
\hline$d g n 1$ & 0.1108 & 0.7542 & 0.1188 & 0.0161 \\
\hline $\operatorname{dgn} 2$ & 0.1108 & 0.7543 & 0.1188 & 0.0161 \\
\hline
\end{tabular}

Thus, according to the transition matrices we are recommended to choose the vertical pattern for 75 times out of 100 times in reconnecting broken edges; the horizontal one 11 times; diagonal1 twelve times; and diagonal 2 one or two times. This result has been applied to Figure 1(C) by selecting candidate edge terminators, where the Bresenham's line drawing algorithm applied using the MATLAB programs [8]. Most broken edges in Figure 1(C) have been reconnected as shown in Figure 2.

\section{$3 \quad$ Edge Linking Based on Regression Heuristics}

Linear regression models the relationship between two variables by filling a linear equation to observed data [11]. This concept is also called line fitting. One variable is an independent variable and the other one is a dependent variable. A linear regression line has an equation of the form $\mathrm{Y}=\mathrm{bX}+\mathrm{a}$, where $\mathrm{X}$ is the independent variable and $\mathrm{Y}$ is the dependent variable. The slope of the line is $b$, and the y-intercept is $a$. The dataset consists of the $\left(\mathrm{x}_{\mathrm{i}}, \mathrm{y}_{\mathrm{i}}\right)$ coordinates of all points where $i$ varies from 1 to $\mathrm{n} ; b$ and a are calculated from the following formulas, shown in equations (5) and (6), using least squares [12] ( $\overline{\mathrm{x}}$ and $\overline{\mathrm{y}}$ are the means of $\mathrm{x}$ and $\mathrm{y}$ values respectively).

$$
\begin{gathered}
\mathrm{B}=\frac{\sum_{t=1}^{n} y t x t-\frac{\sum_{i=1}^{n} y i-\sum_{i=1}^{n} x i}{n}}{\sum_{t=1}^{n}(x t-x)^{2}} \\
\mathrm{a}=\bar{y}-\mathrm{b} \bar{x}
\end{gathered}
$$

In this edge linking method, the dataset for the regression analysis includes the coordinates of all points in an edge component. Using line fitting, we can draw a line through the whole edge segment. This line can show the tendency of the whole object. In Figure 3, an example of the line fitting is illustrated. The dataset resembles the points on a coastline edge component. The line through the points shows the directionality of the whole dataset and gives general information about the whole object.

In the proposed edge linking algorithm, the value of parameters $a$ and $b$ of the linear regression line are needed to be calculated for all objects that are not closed (objects that have endpoints); and $b$ is going to be the slope of the line so that the angle of this line with the positive $\mathrm{x}$ axis is going to be the $\tan ^{-1}$ (slope), which needs to be calculated as well. 


\subsection{Searching for Candidates}

An endpoint is selected from the endpoints set and it is going to be the source endpoint of a linking path. The Euclidean distance needs to be calculated for all of the endpoints other than the selected source. If the distance is less than 10, the angle of the possible line between the source and the endpoint under investigation that can be a possible target endpoint is calculated by the equations (7) and (8). Threshold of the distance is 10 because we do not want to link endpoints too far away each other.

$$
\begin{aligned}
\text { LinkSlope } & =\frac{y_{\text {target }}-y_{\text {source }}}{x_{\text {target }}-x_{\text {source }}} \\
\text { LinkAngle } & =\tan ^{-1}(\text { slope })
\end{aligned}
$$

The position, distance and angle with the positive $\mathrm{x}$ axis for all of the candidate target endpoints are saved to be used in the next step.

\subsection{Decision Making Process}

There is a simple cost function which is based on the distance and the directionality and it can help the decision making process. The best target endpoint from the candidates list is the endpoint which is closer to the source endpoint and the orientation of the link to that endpoint is closer to the orientation of the whole source component. This means that the line which is going to be drawn after the source endpoint is compatible with the line connected to the source.

$$
\Omega=\alpha * \text { Distance }+\beta * \text { IsourceObjectTrendLineAngle }- \text { LinkAnglel }
$$

Any candidate endpoint that generates the minimum value for $\Omega$ is going to be selected as the proper target endpoint for the linking. The $\alpha$ and $\beta$ are weights that are selected empirically. Based on the extensive tests being performed upon the edge result of the satellite coastline images, the chosen values for $\alpha$ and $\beta$ are respectively 1 and 3. In Table 1, various $\alpha, \beta$ values and their effects on the result of edge linking process are shown. It can be concluded that the ratio of the number of wrong links to the total number of links is smaller for $\alpha=3$ and $\beta=1$. The Table 1 is demonstrating the effect of edge linking process on Figures 1(C).

There are two important conditions that need to be considered in this process. The first is to check whether or not the source component has any other source endpoint which is more suitable for this linking. In the source component there may be more than one endpoint. Therefore all of them need to be checked to see if they are more appropriate matches for the selected target endpoint or not. If the $\Omega$ value for any of them is smaller than the $\Omega$ value of the first selected source endpoint, then the previously selected source endpoint needs to be discarded. The edge linking process is then followed by searching a next candidate and to restart the process with the next endpoint. Another condition to check is if any crossing line is occurred due to this linking. The coastlines are continuous lines and a crossing coastline is meaningless. If it is found, then connecting these selected endpoints will result in passing another 
edge which is already in the image; it means that a crossing line is happened. In this instance, the source end point has to be discarded. The edge linking process is then followed by the Search for Candidates procedure, not shown in the paper.

Table 1. Evaluation of edge linking applied to Figure 3 (US)

\begin{tabular}{cccc}
\hline $\boldsymbol{\alpha}$ & $\boldsymbol{\beta}$ & \# of wrong links & Total \# of links \\
\hline 4 & 1 & 4 & 34 \\
3 & 1 & 4 & 35 \\
2 & 1 & 5 & 36 \\
1 & 1 & 5 & 36 \\
\hline
\end{tabular}

\section{Experimental Results}

The Bresenham's Algorithm is used for drawing lines and circular arcs in computer graphics. We are interested in its line drawing methods for the edge linking problem. It uses integers and only needs addition and subtraction operations that are very cheap operations in standard computer architecture. This algorithm determines which pixel should be plotted to draw a straight line between two given points [13], [14]. The following is the summary of the Bresenham's line drawing method. The explicit equation for drawing a line is $y=m x+B$, and its implicit equation is $F(x, y)=a x+$ by $+\mathrm{c}=0$. After applying the Bresenham's line drawing algorithm the two selected endpoints (source and target) need to be discarded to prevent investigating them on the next iterations. The edge linking process is then followed by the Searching for Candidates step.

In the Markov chain based edge linking method, the direction of the next connected line is determined by the current state of the Markov chain; and the probability of the next state is chosen upon the Markov chain matrix. We can enlarge this window and detect the directions of the line connected to the previous pixel of source endpoint. It is a line of three-pixel length and it consists of two lines with different directions such as vertical-vertical, vertical-horizontal, vertical-dgn1, vertical-dgn 2 and etc. Using a new scanning window, one can find the probability of the position of the two adja-cent pixels exactly after the source endpoint. As seen in Figure 2, the Markov Chain based method reconnected the broken lines well, but it could not keep up with its original coastline image; some portions of the coastlines were created randomly because the Markov method provides the overall tendency of those broken lines but not easy to predict well upon local connectivity; and then the comparison of its Figure 2 to Figure 3 tells the obvious differences.

In applying the regression based heuristic approach, the effect of the $\alpha$ and $\beta$ values in the correct decision making process is very important to reconnect the broken edges of Figure 1 successfully; and its result images are shown in the Figure 3: A-C. After applying the Sobel edge detection to the US East coast image of Figure 1(A), the edge linking process is started. The proposed regression based algorithm decides the correct target endpoints very well with $\alpha=3$ and $\beta=1$, as shown in Table 1 and 
Figure 3: A-C. Note that when the value of $\alpha$ has been changed to 4 , then the heuristic function generates more wrong decisions in reconnecting the broken edges. The following images show the final result of edge linking method with $\alpha=3$ and $\beta=1$ on US East coast image in Figure 3. In our proposed method using regression based heuristic, we check the directionality of the whole edge segment by regression analysis and this approach results in detecting more appropriate target endpoints than the Markov chain method.
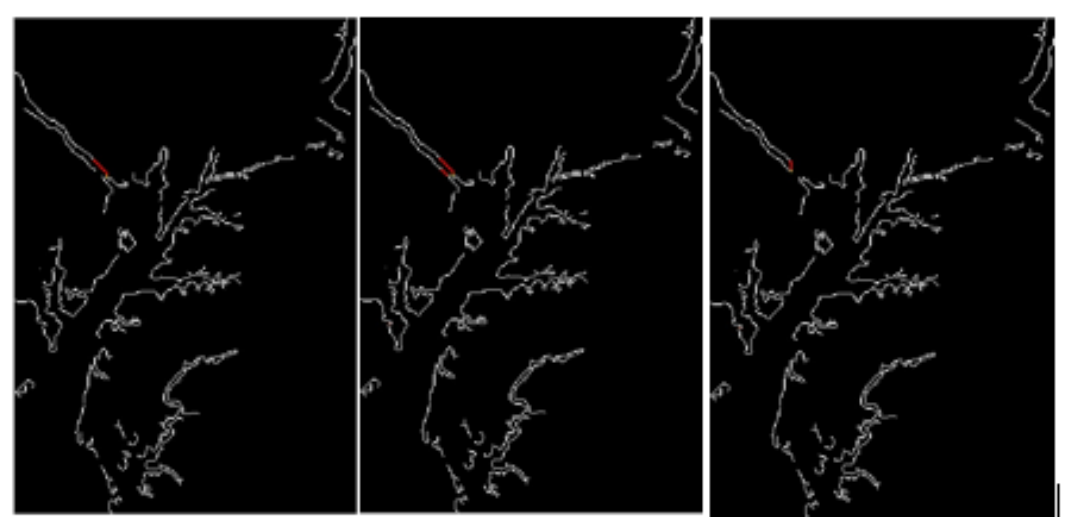

Fig. 3. Starting from the first row left (A), middle (B), and right (C). (A) First correct link with $\alpha=3$ and $\beta=1$, (B) Second correct link with $\alpha=3$ and $\beta=1$, (C) Wrong link with $\alpha=4$ and $\beta=1$.

\section{Conclusions}

In this paper the two edge linking methods of using the Markov Chain and the regression based heuristic function have been proposed and implemented upon the East Coast image of Figure 1. The Markov chain method were trying to reconnect broken edges of a coastline images to provide pattern analysis upon the images that taken by the satellite camera in different time periods at the same location. There has been Markov chain research on soil conservation [15], on which the textures of soil profiles are quantified. Hence, the Markov transition matrix of the same image in different time periods can be used to figure out any changes of a coastline numerically. As seen in Figure 2, the Markov chain has reconnected the broken edges well, however it could not reconnect them all properly; so that there should be more study on how to improve the reconnection ratio using Markov Chain. The regression based heuristic function has been more successful in reconnecting those broken lines of Figure 1; and its reconnections are shown in Figure 3. The heuristic function has reconnected the broken lines more properly where $\alpha=3$ and $\beta=1$ are applied; and it has shown of its proper reconnection of the broken lines than the Markov chain applied to the coastline images. The regression based approach signifies more on the local conditions while the Markov on the global conditions. 


\section{References}

1. Liu, H., Jezek, K.: Automated Extraction of Coastline from Satelite Imagery by Integrating Canny Edge Detection and Locally Adaptive Threshold Methods. International Journal of Remote Sensing 25(5), 937-958 (2004)

2. Guariglia, A., Buonamassa, A., Losurdo, A., Saladin, R., Trivigno, M., Zaccagnino, A., Colangelo, A.: A Multisource Approach for Coastline Mapping and Identification of Shoreline Changes. Annals of Geophysics 49(1), 295-304 (2006)

3. Ryan, T., Sementilli, P., Yuen, P., Hunt, B.R.: Extraction of Shoreline Features by Neural Nets and Image Processing. Photogrammetric Engineering and Remote Sensing 57, 947955 (1991)

4. Sohn, H.-G., Jezek, K.: Mapping Ice Sheet Margins from ERS-I SAR and SPOT Imagenary. International Journal of Remote Sensing 20(15-16), 3201-3216 (1999)

5. Kaur, B., Garg, A.: Comparative Study of Different Edge Detection Techniques. International Journal of Engineering Science and Technology 3(3), 1927-1935 (2011)

6. Kimm, H., Abolhassani, N., Lee, F.: Edge Detection and Linking Pattern Analysis Using Markov Chains. In: Proceedings of the 13th IEEE International Symposium on Advances in Computer and Information Technology, December 3-5 (2013)

7. Neyman, A.: From Markov Chains to Stochastic Games. In: Scholatic Games and Applications. NATO Science Series C, vol. 570, pp. 9-25 (2003)

8. Ghita, O., Whelan, P.: Computational Approach for Edge Linking. Electronic Imaging 11(4), 479-485 (2002)

9. Ching, W., Ng, M.K.: Markov Chains: Models, Algorithms and Applications. Springer (2010)

10. Gonzalez, R., Woods, R., Eddins, S.: Digital Image Processing Using MATLAB, 2nd edn. Pearson Prentice Hall (2004)

11. Montgomery, D., Peck, E., Vining, G.: Introduction to Linear Regression Analysis, 5th edn. Wiley (2012)

12. Weisstein, E.: (1999-2012),

http: //mathworld.wolfram.com/LeastSquaresFitting.html

13. Bresenham, J.: Algorithm for computer control of a digital plotter. IBM Systems Journal 4, 25-30 (1965)

14. Bresenham, J.: A linear algorithm for incremental digital display of circular arcs. Communications of the ACM 20, 100-106 (1977)

15. Li, D., Shao, M.: Simulating the vertical transaction of soil textual layers in north-werstern China with a Markov chain model. Journal of Soil Research, 182-192 (June 2013) 